Radiation in Particle Simulations

R. More, F. Graziani, J. Glosli, M. Surh

November 22, 2010

Radiative Properties of Hot Dense Matter

Santa Barbara, CA, United States

October 11, 2009 through October 15, 2009 
This document was prepared as an account of work sponsored by an agency of the United States government. Neither the United States government nor Lawrence Livermore National Security, LLC, nor any of their employees makes any warranty, expressed or implied, or assumes any legal liability or responsibility for the accuracy, completeness, or usefulness of any information, apparatus, product, or process disclosed, or represents that its use would not infringe privately owned rights. Reference herein to any specific commercial product, process, or service by trade name, trademark, manufacturer, or otherwise does not necessarily constitute or imply its endorsement, recommendation, or favoring by the United States government or Lawrence Livermore National Security, LLC. The views and opinions of authors expressed herein do not necessarily state or reflect those of the United States government or Lawrence Livermore National Security, LLC, and shall not be used for advertising or product endorsement purposes. 


\title{
Radiation in Particle Simulations
}

Richard More ${ }^{1}$, Frank Graziani*, Jim Glosli, Michael Surh

Lawrence Livermore National Laboratory

Livermore, California 94550

(1) Consultant to LLNL

\begin{abstract}
Hot dense radiative (HDR) plasmas common to Inertial Confinement Fusion (ICF) and stellar interiors have high temperature (a few hundred $\mathrm{eV}$ to tens of $\mathrm{keV}$ ), high density (tens to hundreds of $\mathrm{g} / \mathrm{cc}$ ) and high pressure (hundreds of megabars to thousands of gigabars). Typically, such plasmas undergo collisional, radiative, atomic and possibly thermonuclear processes. In order to describe HDR plasmas, computational physicists in ICF and astrophysics use atomic-scale microphysical models implemented in various simulation codes. Experimental validation of the models used to describe HDR plasmas are difficult to perform. Direct Numerical Simulation (DNS) of the many-body interactions of plasmas is a promising approach to model validation but, previous work either relies on the collisionless approximation or ignores radiation. We present four methods that attempt a new numerical simulation technique to address a currently unsolved problem: the extension of molecular dynamics to collisional plasmas including emission and absorption of radiation. The first method applies the Lienard-Weichert solution of Maxwell's equations for a classical particle whose motion is assumed to be known. The second method expands the electromagnetic field in normal modes (planewaves in a box with periodic boundary-conditions) and solves the equation for wave amplitudes coupled to the particle motion. The third method is a hybrid molecular dynamics/Monte Carlo (MD/MC) method which calculates radiation emitted or absorbed by electron-ion pairs during close collisions. The fourth method is a generalization of the third method to include small clusters of particles emitting radiation during close encounters: one electron simultaneously hitting two ions, two electrons simultaneously hitting one ion, etc. This approach is inspired by the virial expansion method of equilibrium statistical mechanics. Using a combination of these methods we believe it is possible to do atomic-scale particle simulations of fusion ignition plasmas including the important effects of radiation emission and absorption.
\end{abstract}

Keywords: particle simulation, radiative processes, fusion ignition, radiation in particle simulation, Lienard-Weichert, electrodynamic normal modes

PAC:

*Corresponding Author: F. Graziani (graziani1@1lnl.gov) 


\section{1.) INTRODUCTION}

The aim of inertial fusion research is to achieve self-sustaining nuclear reactions by compressing DT fuel to high particle density and temperature. To start, or ignite, the nuclear reactions, the ion temperature, $\mathrm{T}_{\mathrm{i}}$, must be in the range of $2-10 \mathrm{keV}$ and the densities must be at least $\mathrm{N}$ times liquid density. The plasma formed in the compression process is further complicated by unequal ion and electron temperatures, impurities of higher $\mathrm{Z}$ material, and by the fusion products such as $3.5 \mathrm{MeV} \alpha$-particles, which cause heating through energy and momentum deposition. Although the DT will be fully ionized the higher $\mathrm{Z}$ material can have bound states that complicate the description of the radiative properties of these plasmas.

In this paper we describe recent work to develop a new tool for particle simulation of hot dense plasmas near the ignition threshold for fusion. At present the specific challenge is to include emission and absorption of radiation in molecular dynamics (MD) particle simulations. In the future we plan to include nuclear reactions and reactionproducts in the simulations.

Inertial fusion plasmas have long been modeled by elaborate calculations using hydrodynamic codes which typically include various forms of energy transport $[15,16]$. The size-scale of these simulations is dictated by the $\sim 10^{-2} \mathrm{~cm}$ size of the target capsules. The hydrodynamic codes assemble theoretical formulas to describe the many underlying physical processes, obtained from kinetic theories of varying complexity and because these kinetic theories come from Landau, Spitzer and other renowned scientists we expect that most of the formulas are good approximations. In practice the hydrodynamic simulations have been compared to a variety of experiments at conditions below the ignition threshold, and have apparently succeeded to describe or even predict experimental results albeit, sometimes with specific calibrations. For example, the range of validity of the radiation-hydrodynamic simulations is not known and the code calculations are sometimes adjusted or normalized to improve their agreement with experiments.

In most cases the theoretical formulas were derived by assuming simplified initial conditions or boundary conditions, or by considering one process at a time, and it is always possible that some unexpected interaction between two microscopic phenomena could make a significant modification in the plasma behavior. In particular, radiative processes are typically studied separately from charged-particle energy-exchange, nuclear reactions and hydrodynamic processes (e.g., diffusive mixing of materials or viscosity effects on flow gradients).

Accurate and detailed experiments on burning plasmas might reveal unexpected couplings between the different processes but even with large new facilities now being prepared, sufficiently detailed experiments will be difficult to perform and diagnose. Despite many years of research on fusion science it is still not true that all the models for 
all the important basic process have been tested with adequate diagnostics at the relevant (extreme high-temperature) conditions.

Fortunately, microscopic particle simulation offers an additional methodology. Particle simulation is based on known fundamental laws: Newtonian particle mechanics under the Coulomb pair-forces, a known coupling to the radiation field and eventually known cross-sections for nuclear reactions. Particle simulation automatically includes any nonlinear interaction between the fundamental couplings, and offers the possibility to see how the "macroscopic" energy-exchange coefficients emerge from the atomic-scale interactions. [1, 2]

Particle simulations do not need to make the typical approximations of kinetic theory, such as predicting three-body correlations by an integral over two-body distribution functions. A particle simulation with enough particles will generate manyparticle correlations without theoretical assumptions and can test the validity of results from kinetic theories. Another important advantage of particle simulation is that the diagnostics are accurate and comparatively inexpensive, although they also encounter questions of signal and noise.

Molecular Dynamics (MD) and Monte Carlo (MC) particle simulations have already provided a great deal of useful information about dense plasmas, especially in the low-temperature strongly-coupled range. However to the best of our knowledge radiation processes have never been included in atomic-scale MD or MC particle simulations.

There should be no doubt that radiation is important to ignition physics. The radiation energy-density may significantly exceed the matter energy-density at temperatures of $2-10 \mathrm{keV}$, and the conversion of plasma energy into radiation is an energy transfer mechanism that will compete with the plasma ion heating. In practice, the conversion is rapid for low-energy photons and slower for high energy X-rays and that difference of rates is important for plasma energetics.

The energy density, U, of ideal black-body radiation in vacuum is:

$$
U=a T^{4}=13.7 \mathrm{MJ} / \mathrm{cm}^{3} T_{k e V}^{4}
$$

where a is the radiation density constant and $T_{k e V}$ is the temperature in $\mathrm{keV}$ units. In comparison the energy-density of ideal-gas DT plasma can be written

$$
\rho E_{\text {mat }}=116 \mathrm{MJ} / \mathrm{gram} \rho_{\mathrm{g} / \mathrm{cc}} T_{\mathrm{keV}}
$$

where $\rho_{g / c c}$ is the mass density in $\mathrm{g} / \mathrm{cm}^{3}$. For fusion conditions the larger numerical coefficient in Eq. (2) does not out-weigh the higher power of temperature in the radiation energy density in Eq. (1). At ignition temperatures, if the radiation is similar to a blackbody distribution, the radiation energy is dominant. Moreover, the equilibrium radiation 
pressure easily exceeds the material pressure. This observation leads to two important questions:

1.) Is the radiation similar to a black-body (Planck) distribution?

2.) Are the ideal-gas estimates sufficiently accurate?

The first question concerns generation of equilibrium radiation in the time before ignition or target disassembly. While the second question concerns non-ideal corrections due to coupling between particles, waves and radiation. Because the equilibrium radiation pressure and energy are large, even modest corrections might play an important role for plasma hydrodynamic behavior.

For pure hydrogen, or DT, plasmas the electron-ion Coulomb energy-exchange is faster than radiation processes. Even when multiplied by the small electron-ion massratio, $\mathrm{m}_{\mathrm{e}} / \mathrm{M}_{\mathrm{i}}$, the Coulomb cross-section exceeds the radiative cross-sections, so the temperature-equilibration between electrons and ions is faster than the generation of radiation. [The energy exchange times are inversely proportional to the cross-sections.] Electron-electron and ion-ion energy exchange are faster still. However, there are several reasons to include radiation processes in the simulation of the plasma evolution. First, the time-scale of interest is the time-scale for ignition. Just as the Coulomb crosssection exceeds the radiative cross-sections, so the radiation cross-sections greatly exceed the fusion cross-sections, and therefore radiative phenomena will be well advanced before fusion self-heating and ignition can occur. Second, including radiation processes is necessary because all experiments involve plasmas with impurities of various atomic species. The elements present will depend on the specific experiment, but whatever the target materials the impurity atomic number $\mathrm{Z}$ and/or ionization state $\mathrm{Z}^{*}$ are necessarily greater or much greater than unity. Since the radiative rates rise with high powers of $Z$ (or $Z^{*}$ ) radiative phenomena are much more important for plasma with impurities than for pure-hydrogen or DT plasmas.

Given these two reasons, we conclude that a treatment of radiative phenomena is essential to a realistic particle simulation of fusion ignition. Since there are no established techniques to perform simulations with both radiation and atomic particle dynamics, we have chosen to explore various algorithms and approaches in search of an appropriate method.

Here we report on the effort to develop an appropriate method by considering four approaches. The first method applies the Lienard-Weichert solution [3] of Maxwell's equations for a classical particle whose motion is assumed to be known (Section 3). The second method expands the electromagnetic field in normal modes $[4,5]$, in this case plane-waves in a box with periodic boundary-conditions, and solves the equation for wave amplitudes coupled to the particle motion (Section 4). The third method is a hybrid molecular dynamics/Monte Carlo, MD/MC method which calculates radiation emitted or absorbed by electron-ion pairs during close collisions (Section 5). The fourth method is a generalization of the third method to include small clusters of particles emitting radiation during close encounters: one electron simultaneously hitting two ions, 
two electrons simultaneously hitting one ion, etc. (Section 6). This approach is inspired by the virial expansion method of equilibrium statistical mechanics.

Each of these four approaches has been implemented in test calculations of various sizes and each offers some insight and poses some difficulties. We want to evaluate these methods to see whether they can obtain the right answer, whether they are computationally practical, and to see whether they offer the generality and elegance that we always wish to find in a new method of plasma modeling.

\section{2.) RADIATION IN PARTICLE SIMULATIONS: GENERAL CONSTRAINTS}

The particle simulations solve many-body classical equations (Newton's laws for Coulomb forces) and the most straightforward coupling to the electromagnetic field (through Maxwell equations) will give a classical description of radiation. Our classical particle simulations must be modified to incorporate the fundamentally quantum mechanical nature of the radiative emission and absorption processes. Ideally, one would like to solve the N-body quantum problem coupled to radiation exactly. However, the Nbody quantum problem without radiation becomes computationally challenging even for modest size problems [21]. Therefore, due to the particle number requirement of hot dense plasmas, the best alternative currently is to find radiation methods that augment the classical MD approach.

As we explore different approaches, we face the difficulty that a complete and rigorous solution of Maxwell's equations for the classical many-particle system would give an incorrect result: instead of the Planck distribution, it would predict the RayleighJeans distribution for the radiation intensity. The Rayleigh-Jeans law has far too much energy in high-energy x-rays, and this would completely invalidate the energetics of the simulation. Therefore, we must introduce some quantum features onto the classical particle dynamics.

A related technical difficulty for simulations with radiation is caused by the technique of quantum mechanical effective two-body potentials (QMEP) which are used in MD simulations to soften the Coulomb interaction between electrons and positive charges at short distances $[17,18,19]$. These potentials are rigorously derived but only for systems in equilibrium. Even with this weakness, a strong practical argument for the use of QMEPs to non-equilibrium systems is that it suppresses recombination in a classical (without radiation) MD simulation. The recombination occurs through the classical version of 3-body recombination: during an electron-ion collision, some energy can be transferred to a distant particle (usually an electron) and that process leads to recombination into boundstates. In classical mechanics the boundstates have no lower limit on their energies, an instability known since the earliest days of quantum mechanics. Emission of radiation adds another recombination mechanism and intensifies this difficulty. 
If we consider a simple collision of one electron with one proton, we know that the exact non-relativistic (quantum) solution of the Schrödinger equation gives the Rutherford cross-section, assuming plane wave states for the electrons. This is exactly the same as the classical cross-section for the same potential. (The Rutherford crosssection neglects relativistic effects such as spin, spin-orbit interaction, retardation, magnetic and smaller relativistic effects.) A characteristic of the QMEPs is that the long-range behavior of the potential is unchanged. This means that many plasma collective phenomena (screening, plasma waves, Coulomb energy exchange, etc.) are also largely unchanged. Unfortunately, due to the softening of the Coulomb barrier for short distances, the QMEP approach changes the behavior for collisions with large scattering angles and those collisions are most important for emission or absorption of radiation, especially for high-energy ("hard") photons. In our work, we have searched for a way around this difficulty and the algorithm described in Section 5 below offers an alternative to the QMEP method.

The algorithm described in Section 5 treats the close collisions by a different method from that used for the main MD simulation. When an electron and an ion approach to within a small distance (typically $\sim 0.2 \AA$ ) they are treated as a two-body system and their collision is described by matching the current position and velocity to the classical Coulomb-scattering trajectory for the motion of the pair. This solution is used until they again separate. Since this part of the orbit is entirely governed by 2-body forces the 3-body recombination is automatically suppressed. We also can permit emission and absorption of bremsstrahlung radiation during the close collision, controlled by a Monte Carlo algorithm described in Section 5, and this leads to a simulation that behaves in a satisfactory manner. However, there are questions or difficulties for this method and we discuss them in depth in this paper.

Quite generally, adding radiation to a particle simulation involves both a conceptual challenge (e.g. what is the meaning of photons in a classical context?) and challenges related to practical implementation in a code. Here we provide a list of difficulties, to be discussed in more detail on Sections 3 through 7:

a.) Stochastic Evolution. The basic quantum nature of emission/absorption appears inconsistent with deterministic molecular dynamics (MD) time-evolution. That is, quantum mechanics predicts probabilities for emission and absorption but does not give a deterministic prediction which process occurs in a given encounter.

b.) Time-scales. The generation of radiation is slow for the electron-proton system, so the simulation must be followed for many time-steps in order to see the appearance of a significant radiation field. The electron time-step is typically less than $10^{-19} \mathrm{sec}$ but copious radiation only appears after $\sim 10^{-10} \mathrm{sec}$. Of course our ultimate goal is to carry the simulations for still longer times $\left(\sim 10^{-8} \mathrm{sec}\right)$ to see fusion processes. All the radiative rates rise rapidly with $\mathrm{Z}$ so plasmas with impurities are more active in producing 
radiation. The radiative rates can be artificially increased in a simulation by deliberately increasing the cross-sections (both absorption and emission) by a multiplicative factor to see how the approach to plasma-radiation equilibrium will proceed; however this is not quite equivalent to scaling the time variable because it does not affect all processes in the same way.

c.) Sampling. The expected number of photons is only comparable to the particle number at high temperatures. In a $1000 \AA^{3}$ simulation box there will only be a few photons at $\mathrm{kT}_{\mathrm{R}} \sim 1 \mathrm{keV}$ and this will make a signal-to-noise problem for our simulations. However, at temperatures above $\sim 3 \mathrm{keV}$ this difficulty is greatly reduced.

d.) Fundamental data. We have good approximate rates for radiative processes involving one atom (ion) in a spherically symmetric environment, but do not have corresponding rigorous quantum calculations for non-spherical or multicenter clusters. To analyze emission from such systems we will use classical or hybrid semiclassical methods. It would be very useful if we had rigorous quantum calculations to estimate the accuracy of our methods.

Early in this work we were concerned that the technique of simulating a number of particles confined in a computational box could lead to some unrealistic coupling to radiation. For example, if the particles are confined so they bounce from the walls of the computational box, these wall reflections correspond to abrupt accelerations: won't the accelerations cause spurious or unphysical radiation? Instead if the particles are subject to periodic boundary conditions, then when an electron leaves the box on the right wall it is instantaneously re-injected on the left: in effect a sudden jump in position. Does that make some kind of Cerenkov emission? Careful study has shown these problems do not arise if the simulation is performed in a sensible way, such that the electromagnetic field obeys the same boundary conditions as the particles. For example, if we used reflecting walls, the abrupt acceleration would occur at a zero of the electromagnetic wave-field and would therefore not couple to radiation. (Periodic boundary-conditions are discussed more thoroughly in sections 3 and 4.)

Finally, both classical and quantum electrodynamics calculations can lead to divergent predictions $[3,4,5]$. In general the divergences affect only the calculation of higher-order processes, e.g., electron self-energy or vertex corrections. Although there exists a very satisfactory method, the S-matrix renormalization theory, to remove the divergences, at least for few-particle collision processes, it is not clear how these methods work for a statistical ensemble of dense particles continuously interacting with radiation. Due to this uncertainty, as we discuss the possible methods, we point out the possibility of problems with radiation reaction, self-interaction, etc. Divergent integrals do not occur in our work because we never consider an infinite number of normal modes; a high-frequency cutoff is artificially imposed by computer limitations. As is true for all particle simulations, our simulations make no effort to obtain results that are covariant. The radiation algorithm described in section 5 does not require any specification of a gauge and there is no specific consideration of gauge invariance. The MD algorithms 
applied here rely on forces acting on particles that are mediated by gauge invariant electric and magnetic fields.

\section{3.) LIENARD-WEICHERT POTENTIALS}

Classical one component particle, OCP, simulations assume instantaneous Coulomb interactions between charged particles. [1] In most cases the simulations are performed for $\mathrm{N}$ particles in a simulation box embedded in an environment modeled by periodic boundary conditions. It is known that the technique of periodic continuation leads to rapid convergence of the free energy and other equilibrium properties with the size of the simulation box. [2]

To introduce radiation into such a classical MD particle simulation, classical electrodynamics offers two straightforward approaches. One can replace the instantaneous Coulomb interaction by the retarded potentials (Lienard-Weichert potentials) for the electromagnetic field of classical point charges following known trajectories.[3] Alternatively, as discussed in Section 4, the classical electromagnetic field can be expanded in normal modes. In this case Maxwell's equations give differential equations for the field amplitude coefficients. In this section we discuss the Lienard-Weichert method. One purpose of the discussion is to underline that these two methods give the same results, with different degrees of convenience and flexibiity.

We have written a simple computer code to evolve the motion of a few-particle system replacing the instantaneous Coulomb interaction with forces obtained from the Lienard-Weichert electric and magnetic fields. In doing this we encounter interesting issues concerning all MD plasma simulations.

First, the usual Coulomb MD simulation neglects the magnetic forces between moving particles. For the ion OCP this neglect is clearly justified, but for simulations of electron motion in hot plasmas the approximation is debatable, as the magnetic forces are of order $\mathrm{v} / \mathrm{c}$ relative to electrical forces. This parameter is not small in a fusion ignition plasma. For example, at an electron temperature of $2 \mathrm{keV}$ the ratio of the particle velocity, $v$, to the speed of light, $\mathrm{c}$, is $v_{e} / c \sim .11$, illustrating that the magnetic forces are already a $10 \%$ correction.

Second, the usual periodic boundary conditions are inconsistent with relativistic causality: these boundary conditions assume instantaneous correlations between particles separated by large distances ignoring the requirement that these correlations can only propagate at speeds less than, and for plasma waves much less than, the speed of light. This is an objection in principle, but it is not clear that artificial long-range correlations have a significant effect on thermodynamic properties in the limit of large N. 
Third, the strong electric fields and large energy density of radiation are neglected in the usual simulations. Using the Planck formula we calculate the RMS microfield of equilibrium black-body radiation, $E_{\text {Rad }}$ :

$$
E_{\text {Rad }}=1.210^{10}\left(\frac{\text { Volt }}{\mathrm{cm}}\right)\left(\frac{T_{R}}{\mathrm{keV}}\right)^{2}
$$

where $T_{R}$ is radiation temperature. At temperatures in the $\mathrm{keV}$ range, this electric field exceeds the Coulomb fields of neighbor ions even at typical inertial fusion densities. Of course $\mathrm{E}_{\mathrm{Rad}}$ is rapidly oscillating and the electron response to a rapidly oscillating field is only a small "quiver velocity"

$$
\delta v_{r a d} \sim e E /(m \omega)
$$

This quiver velocity is evidently less than the electron thermal velocity because most of the black-body photons have high frequencies. However, since $\langle\omega\rangle \sim T, \delta v_{\text {rad }}$ grows proportional to $T$ while the thermal velocity is $\sim \sqrt{ } T$. Additional study and verification are probably needed to ensure that the large field of Eq. (3) plays no role in atomic or nuclear processes.

\section{Lienard-Weichert potentials}

For calculations by the Lienard-Weichert (LW) method, an electron's position and velocity, $\boldsymbol{r}_{p}(t)$ and $\boldsymbol{v}_{p}(t)$, are assumed to be known, and we use the LW solution of Maxwell equations for the $\boldsymbol{E}, \boldsymbol{B}$ fields generated at a field point $\boldsymbol{r}, t .[3]$ These fields are simply expressed in terms of the retarded time $t_{r e t}$ and retarded distance $\boldsymbol{R}_{\mathrm{r}}$ defined by:

$$
R_{r}=r-r_{p}\left(t_{r e t}\right) \quad R_{r}=\left|r-r_{p}\left(t_{r e t}\right)\right|=c\left(t-t_{r e t}\right)
$$

Here we use the same notation for vector and scalar distance, e.g., $R_{r}$ in the line above; the notation can easily be corrected. In the usual Lorentz gauge the fields are obtained from scalar and vector potentials,

$$
\phi=\frac{q}{\left(R_{r}-\frac{v \cdot R_{r}}{c}\right)}=\frac{q}{\mathrm{R}} \quad \vec{A}=\frac{q}{c\left(R_{r}-\frac{v \cdot R_{r}}{c}\right)}=\frac{q}{c} \frac{\vec{v}}{\mathrm{R}}
$$

A useful scalar distance $R$ is defined by:

$$
\mathrm{R}=\left(R_{r}-\frac{\vec{v} \cdot \vec{R}_{r}}{c}\right)
$$


When appropriately differentiated the potentials give the electric and magnetic fields at the field point $r, t$ :

$$
\begin{aligned}
& \vec{E}=\frac{q}{\mathrm{R}^{3}}\left(1-\frac{v^{2}}{c^{2}}\right)\left[\vec{R}_{r}-\frac{\vec{v}}{c} R_{r}\right]+\frac{q}{\mathrm{R}^{3} c^{2}} \vec{R}_{r} \times\left(\left[\vec{R}_{r}-\frac{\vec{v}}{c} R_{r}\right] \times \vec{a}\right) \\
& \vec{B}=\frac{\vec{R}_{r}}{R_{r}} \times \vec{E}
\end{aligned}
$$

Such fields are produced by each particle in the plasma. Each particle is subject to the forces produced by the other particles (there is no self-interaction). The formula for the electric field consists of two terms with different dependence on radius. The first term $\left(\sim 1 / R^{2}\right)$ is a modified Coulomb field while the second term $(\sim 1 / R)$ is also proportional to the acceleration $\boldsymbol{a}$ evaluated at the retarded time, and leads to the emission of radiation.

To use the Lienard-Weichert fields in a simulation we save the classical trajectories $\boldsymbol{r}_{j}(t), \boldsymbol{v}_{j}(t)$ for each particle. We have this data from each previous time-step, and store the data for $\sim 10^{5}$ time-steps. When we want to calculate the force on particle $j$, we search the trajectories for each other particle $k$ to find the retarded time for the pair $(j, k)$. We find time-steps that bracket the retarded time and interpolate. To speed-up the search, it is useful to save a pointer to the previous retarded time; the $t_{\text {ret }}$ only increases by approximately the time-step $d t$, so the previous value of $t_{\text {ret }}$ is a good first guess.

At the beginning of the simulation $(t=0)$ we launch the particles with random positions and velocities sampled from an appropriate Maxwellian. For the first time steps we extrapolate to earlier times $(t<0)$ to find the retarded times and distances; we do this assuming straight-line motion before $t=0$. Since there is no acceleration at these unphysical times, no radiation is produced. For nearby particles the retarded time is very "recent", i.e., occurs at a time after the end of the last previous time-step. For these cases an extrapolation procedure is needed rather than simple interpolation.

For the LW method, we sum over the N particles in the simulation box and also over a finite number of image charges for each particle, ignoring the difficult question about convergence with the number of images. The retarded times of the image charges grow with distance and so the simulation must run for a certain length of time before image-charge location data becomes available. We do not start to accumulate data for the thermodynamic averages until the calculation has progressed long enough so that dynamical data (calculated with accelerations) has become available for all particles and their images.

We use electrical forces from the past positions of the image-charges but their current positions are assumed to be exactly correlated with the current positions of the $\mathrm{N}$ particles in the simulation box. This is the causality issue. The image charge's past positions affect the motion of the charges in the box after a time-delay of $\Delta t \sim R / c$, but the 
current positions of the box particles instantaneously determine the current positions of the image particles. The round-trip influence time is then $R / c$ instead of $2 R / c$. One might worry that this artificial correlation acts like a "Maxwell Demon" to perturb the entropy of the simulation; deeper study would be needed to assuage this concern. This is an issue, not just for the Lienard-Weichert simulation but for any particle simulation using periodic boundary conditions. In fact, for pure Coulomb potentials, as is usual in MD simulations, the round-trip influence time is zero.

The LW potentials give pair-forces between the electrons and ions. In principle the same formulas also predict the EM field throughout the volume of the plasma simulation box. The LW formulas enable us to distinguish emitted radiation from fields attached to the particles (Coulomb $+v x B$ magnetic fields) but Fourier analysis would be required to extract the radiation spectrum.

The LW method has difficulty describing a pre-existing radiation field, for example in a plasma receiving radiation emitted by a high-temperature source. In order to describe such radiation the simulation would have to carry additional variables such as the amplitudes of the various normal modes of the radiation field. If this were done one might as well use the method of the next section.

The most important defect of the LW method is that it is not quantum mechanical. If we Fourier analyze the LW fields $\boldsymbol{E}(\boldsymbol{r}, t), \boldsymbol{B}(\boldsymbol{r}, t)$ the best result we can hope for is the incorrect Rayleigh-Jeans spectrum. We did not continue our LW simulations long enough to see the onset of this problem.

The LW method is clearly an $\mathrm{O}\left(\mathrm{N}^{2}\right)$ algorithm - we find the force on each particle by a summation over contributions from each other particle - and this limits the number of particles that can be treated. We do not have a generalization of the Ewald summation technique to include retardation and magnetic effects, and have underlined the causality difficulties with such a generalization. Figure 1 shows the simulations results of two electrons and two ions interacting via Coulomb and radiation fields. The quantity plotted on the sphere is the emission through a large sphere surrounding the simulation box. The shading denotes the magnitude of the normal component of the Poynting vector. The dipole nature of the emission spectrum is clearly indicated by the shading. As the electrons move through the system, the shading moves around the ball but always in a way such that the intensities along two opposite poles are zero. The emission has an instantaneous dipole pattern. 


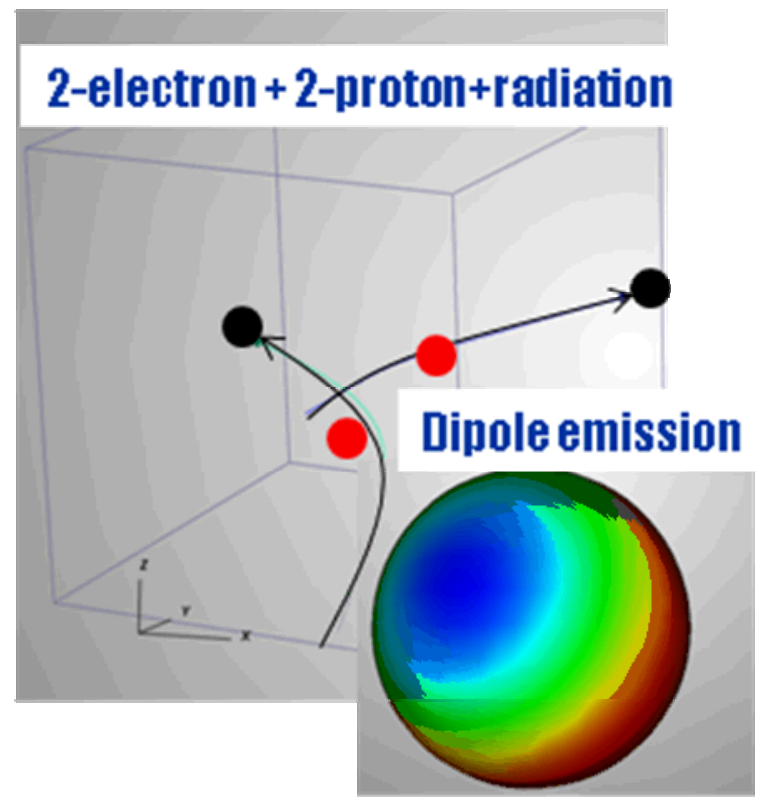

Figure 1: MD plus radiation results for two electrons scattering off of two ions. The sphere represents a unit sphere surrounding the simulation box and the colors refer to the normal component of the Poynting vector.

\section{4.) NORMAL-MODE EXPANSION OF EM FIELD}

In the normal-mode method the classical electromagnetic field is expanded in a set of propagating waves that obey periodic boundary conditions in some rectangular box. The normal-mode amplitudes change due to absorption and emission by the particles in the simulation box. Since the initial normal-mode amplitudes can be chosen arbitrarily we can simulate plasmas with arbitrary initial radiation spectra with this method. It is most natural to define these normal modes in the same simulation box used for the particles. The advantage of this choice will be discussed below.

The technique requires many normal modes. For example, using 200 wavevectors in each spatial direction yields $8 \times 10^{6}$ normal modes. The largest allowed wavevector $\mathrm{k}$ determines the spatial resolution inside the simulation box. The time-step in the simulation should resolve the highest photon frequency $\omega$ unless we adopt some method to integrate analytically over short time intervals. For example, we can use a $(100 \AA)^{3}$ box with $8 \times 10^{6}$ normal modes where the high frequency modes have a wavelength of about $\sim 1 \AA$, consistent with our interest in $\mathrm{h} v \sim 10 \mathrm{keV}$ photons. 


\section{Normal-mode expansion for classical electrodynamics:}

It is natural to write the equations for the normal-mode expansion in a notation close to non-relativistic Coulomb-gauge quantum electrodynamics [4, 5]:

$$
\begin{gathered}
A(\vec{r}, t)=\sum_{k, \lambda} \hat{e}_{k, \lambda}\left(\frac{2 \pi \hbar c^{2}}{\omega_{k} V}\right)^{1 / 2}\left[a_{k, \lambda} e^{i \vec{k} \cdot \vec{r}}+a_{k, \lambda}^{+} e^{-i \vec{k} \cdot \vec{r}}\right] \\
\frac{\partial A(\vec{r}, t)}{\partial t}=\sum_{k, \lambda} \hat{e}_{k, \lambda}\left(\frac{2 \pi \hbar c^{2}}{\omega_{k} V}\right)^{1 / 2}\left(-i \omega_{k}\right)\left[a_{k, \lambda} e^{i \vec{k} \cdot \vec{r}}-a_{k, \lambda}^{+} e^{-i \vec{k} \cdot \vec{r}}\right]
\end{gathered}
$$

In these equations, $\boldsymbol{k}$ denotes a photon wave-vector and $\mathbf{e}_{\mathrm{k} \lambda}$ is the polarization vector of the mode defined by $\boldsymbol{k}, \lambda(\lambda=1,2$ identifies the polarization state). The notation

$$
C_{k}=\left(\frac{2 \pi \hbar c^{2}}{\omega_{k} V}\right)^{1 / 2}
$$

is often used for the coefficient in Eqs. $(12,13)$. In the quantum theory, $a$ and $a^{+}$are field operators but in the classical case they are simply complex numbers; $a_{k \lambda}(t)=$ complex field strength, $a^{+}$is its complex conjugate. Here $V$ is the volume of the quantization box and $\omega=c k$ is the photon frequency.

This expression for the vector potential assumes the Coulomb (transverse) gauge, expressed by the condition $\boldsymbol{k} \cdot \boldsymbol{e}_{k \lambda}=0$. The functional forms for the vector potential and its derivative already guarantee that the electrical and magnetic fields will satisfy three of the Maxwell equations. The fourth Maxwell equation is satisfied if the field amplitudes $a_{k \lambda}$ obey an equation for the coupling to the particles.

$$
i \hbar \frac{\partial a_{k \lambda}}{\partial t}=\hbar \omega a_{k \lambda}-\sum_{p} \frac{q}{c} C_{k}\left(\vec{v}_{p} \cdot \hat{e}_{k \lambda}\right) e^{-i k \cdot r_{p}(t)}
$$

From Eq. (15) we can easily derive an equation for the photon number $n_{k}=a_{k \lambda}{ }^{+} a_{k \lambda}$,

$$
\hbar \omega \frac{\partial n_{k \lambda}}{\partial t}=q\left(\vec{v}_{p} \cdot \hat{e}_{k \lambda}\right) \frac{i \omega}{c} C_{k}\left(a_{k, \lambda} e^{i k \cdot r_{p}(t)}-a_{k, \lambda}^{+} e^{-i k \cdot r_{p}(t)}\right)
$$

A higher-order coupling $\left(\sim \mathrm{A}^{2}\right)$ is omitted here. For this normal-mode expansion one has separated transverse and longitudinal contributions to the fields, their energies and to the energy flux. The technical details of the separation are explained in several references (e.g., Kroll [4], Cohen-Tannoudji [5]).

Given the vector potential and its derivatives, the radiation electric and magnetic fields are calculated by 


$$
\begin{array}{ll}
E_{k, \lambda}=\hat{e}_{k \lambda} \frac{i \omega}{c} C_{k}\left(a_{k, \lambda} e^{i k \cdot r}-a_{k, \lambda}^{+} e^{-i k \cdot r}\right) & E=-\frac{1}{c} \frac{\partial A}{\partial t} \\
\vec{B}_{k, \lambda}=i \vec{k} \times \hat{e}_{k \lambda} C_{k}\left(a_{k, \lambda} e^{i k \cdot r}-a_{k, \lambda}^{+} e^{-i k \cdot r}\right) & B=\nabla \times A
\end{array}
$$

To these fields must be added the instantaneous Coulomb field between the point charges. Corrections to the instantaneous Coulomb field due to retardation and relativistic distortion of the $1 / r^{2}$ field are obtained through Eq. (17) from Eq. (15). These corrections can be thought of as due to "bound photons" carried by a moving charged particle.

For the case in which there is no coupling to particles, the equation of motion, Eq. (15), for $a_{k \lambda}$ has the simple free-field solution $\mathrm{a}_{k \lambda} \sim e^{-i \omega t}$. When there is a coupling, the amplitude changes corresponding to emission and absorption. In the classical theory these are distinguished by the relative phases of the terms in Eq. (15). Equation (15) is easily solved for the simplest case of coupling to a particle that moves with constant velocity $v$. The result agrees exactly with the appropriate Coulomb-gauge version of the Lienard-Weichert solution for the same case.

We can evolve the normal-mode amplitudes using Eq. (15) and then reconstruct the local electric and magnetic fields. This method gives the classical electromagnetic theory in a form closely parallel to nonrelativistic quantum electrodynamics. The classical equations (12 - 15) contain Planck's constant but only as a normalization of the field amplitudes $a_{k} \lambda$; it can be entirely removed by a change of this normalization. The particle motion is governed by the total electric and magnetic fields at the location of the particle, so the particles respond to any existing radiation.

\section{Test-code for the normal-mode method}

As mentioned above, our calculations include $8 \times 10^{6}$ normal modes, which define the spatial resolution. The time-step must be small enough to resolve changes in the particle velocity including high-frequency perturbation by the x-ray photons $v_{p}(t)$. The electric and magnetic fields reconstructed from the normal-mode amplitudes, $\boldsymbol{E}(\boldsymbol{r}, t)$ and $\boldsymbol{B}(\boldsymbol{r}, t)$, contain some numerical noise because of the imperfect spatial resolution. Despite this noise it is clear that the results agree with $\boldsymbol{r}$-space Lienard-Weichert calculations; in particular, the distortion of the static Coulomb field due to the particle motion, and the magnetic field are found to be essentially the same.

For the normal-mode expansion we find (both numerically and analytically), that if the radiation normal modes are defined in the same box used for the particles, then the fields (near field, emitted radiation) generated by the particles move as if in the periodically extended system. 
For example, the $\mathrm{O}\left(v^{2}\right)$ corrections to the Coulomb potential remain attached to a particle as it exits one side of the simulation box and re-enters the opposite side. It is especially interesting that part of the radiation correction precedes the electron and jumps across the box before the electron itself. The distorted Coulomb field follows when the particle crosses the cell boundary because we use periodic boundary conditions with the same quantization box for the radiation as used for the particle simulation. The corrections to Coulomb's law obtained from the normal mode method agree exactly with the distortions described by the Lienard-Weichert formulas, when evaluated for the same particles undergoing the same motion.

The normal-mode method automatically includes absorption and emission processes. Absorption occurs because coupling to the high-frequency electric field is contained in the particle equation of motion as well as in the radiation equation.

It might be possible to sample and interpolate for the normal modes, without keeping so many degrees of freedom. It is reasonable to expect an isotropic plasma will create an isotropic radiation spectrum (apart from fluctuations) and that expectation could be forced onto the algorithm with a consequent loss of information about interference effects. We have not yet attempted this type of calculation.

There is an interesting question about self-interaction. In the LW method, all the interactions between particles are explicit pair-interactions. This includes cases in which a photon is emitted, propagates some distance, and then is absorbed later. However, we do not allow any self-interaction. Interaction of a particle with its periodic image is included in the calculations. In the normal mode approach, each particle changes the general radiation field that then can interact back with the same particle, so there is a question of consistency. In fact in the simulations any radiation field generated by a particle leaves the vicinity of that particle at the speed of light, and only returns to interact with the same particle after reflection from the box boundary. Our current simulations do not exhibit any problem from self-interaction.

These simulations are entirely classical (classical particles and a classical electromagnetic field). They necessarily produce an incorrect result for the highfrequency electromagnetic field, as they must relax to the Rayleigh-Jeans distribution instead of the Planck distribution for the blackbody radiation. For fusion plasmas the Rayleigh-Jeans distribution would be seriously incorrect. For the long-wavelength part of the electromagnetic spectrum the Rayleigh-Jeans law is not significantly incorrect. So our ultimate algorithm may use the normal mode equations for this part of the radiation field.

A fully-quantum simulation would follow a rapidly-increasing family of copies of the system with different numbers of photons in different photon modes and must permit transitions including interference between these copies. A simple estimate of the number of states makes this approach seem impractical. For example, to follow states with 0,1 and 2 photons each for $10^{7}$ photon modes would require carrying $3^{10,000,000} \sim 10^{3,000,000}$ amplitudes. 
Finally there is an important computational issue regarding the scaling of the normal mode method with the number of particles or size of the system. We imagine comparing systems of constant particle density in larger and larger simulation boxes. The number of particles $N_{p} \sim L^{3}$, where $L$ is the edge of the box. To obtain a constant spatial resolution, comparable to the particle separation the normal mode method requires us to carry more normal modes for the larger box: basically the number of normal modes also scales as $L^{3}$. Since each normal mode must interact with each particle, this is also an $N_{p}{ }^{2}$ algorithm, like the LW method.

\section{5.) BINARY ENCOUNTER EMISSION/ABSORPTION MODEL}

Given the discussions of the previous methods it is clear we must include quantum effects in the simulation, at least in a semiclassical form, in order that the simulations satisfy the common-sense requirement that they relax toward a Planck spectrum for the radiation.

Apparently the simplest way to accomplish this follows Einstein's original discussion of detailed balance [6]: the probabilities of absorption and emission during a collision are required to be respectively proportional to expressions of the form

and

$$
\text { absorption } \sim f\left(E_{l}\right) v_{l} B n_{v}
$$

$$
\text { emission } \sim f\left(E_{u}\right) v_{u} B\left(n_{v}+1\right)
$$

respectively, where $f(E)$ is the electron distribution function for lower and upper electron energy-states, denoted by subscripts $l, u ; v_{l}, v_{u}$ are the approach velocities; $n_{v}$ is the number of photons per normal mode; and, $B$ is a coefficient which must be equal for two processes related by time-reversal. We use the symbol $B$ to suggest the Einstein $B$ coefficient, which refers to line transitions, but in our case $B$ is an appropriate average of the bremsstrahlung cross-sections and depends on electron and photon energies.

The pair of electron states related by detailed balance have different energies $E_{u}$, $E_{l}$ which differ by the energy $h v$ of the photon emitted/absorbed, i.e., $E_{u}-E_{l}=h v$. The one-electron states in the classical simulation have specified position and velocity whose apparent accuracy exceeds the uncertainty limit. With this formulation of the radiative processes we expect that the simulation will drive the two species (electrons, radiation) toward equilibrium with a Maxwell-Boltzmann distribution for the electrons and a Planck distribution for the radiation, and if the coefficient $\mathrm{B}$ is approximately correct the relaxation will occur on an realistic time-scale. When the system reaches equilibrium the rates of emission and absorption will be equal.

We do not have a deterministic algorithm to implement these rates, and instead assume there are probabilities for emission and absorption during electron-ion collisions. 
These probabilities are largest for the strong collisions, i.e., collisions with small impact parameters. In the present code other atomic processes (ionization, excitation, recombination, etc.) are omitted but effort is underway to include these processes using similar methods. The use of probabilities means we will combine molecular dynamics, which is a classical and deterministic simulation, with the Monte Carlo method, which uses random numbers to make choices during the simulation, in a semi-quantum mechanical simulation. To obtain a feasible algorithm we implement the Einstein expressions in Eq. $(19,20)$ for absorption/emission rates using a hybrid molecular dynamics/Monte Carlo (MD/MC) algorithm described here. Our method requires approximations that ignore potentially interesting, but small, effects.

\section{MC/MD Algorithm}

In this approach the radiation field will be represented by an isotropic homogeneous photon spectrum $I_{v}$. In equilibrium $I_{v}$ is expected to be the blackbody function $\mathrm{B}_{v}$ :

$$
B_{v}=\frac{2 h v^{3}}{c^{2}} n_{v}^{0}=\frac{2 h v^{3}}{c^{2}} \frac{1}{e^{h v / k Y}-1}
$$

Here the symbol $n_{v}$ again denotes the number of photons per normal mode of the radiation field. We calculate radiation emitted and/or absorbed in electron-ion collisions and ignore electron-electron or ion-ion collisions, which have much smaller (quadrupole $\sim \mathrm{kT} / \mathrm{mc}^{2}$ ) rates. At present we also ignore Compton scattering although ultimately it will be included.

In this approach we assume the radiation field is isotropic and replace the many normal mode amplitudes by the photon energy distribution. This means that instead of carrying $10^{7}$ normal modes we carry only $\sim 100$ photon energy-bin populations. We make no attempt to represent any spatial variation of the radiation field inside the simulation box, i.e., we are considering electron-photon energy exchange but not radiative transfer.

The MC algorithm for radiation emission/absorption assigns a conditional probability of emitting/absorbing during each electron-ion collision. We convert existing formulas for bremsstrahlung cross-sections into appropriate conditional probabilities while preserving detailed balance. We begin with the Kramers absorption and emission cross-sections.[7] Here we write the cross-sections and show how they are normally used in kinetic theory, i.e., how they generate a number of radiative events $d N$ per volume $d^{3} r$, time $d t$ and photon energy range $d h v$. The notation matches an earlier review article by one of the authors.[8]

Inverse bremsstrahlung absorption is a 3-body reaction (the initial particles are electron, ion and photon), so the absorption cross-section $\sigma^{a b s}$ has units $\mathrm{cm}^{5}$. The Kramers form for $\sigma^{\text {abs }}$ is 


$$
\sigma^{a b s}=\frac{8 \pi^{3}}{3 \sqrt{3}} Z^{2} a_{o}^{5}\left(\frac{e^{2} / a_{o}}{h v}\right)^{3}\left(\frac{e^{2} / a_{o}}{\varepsilon}\right)
$$

If $\sigma^{a b s}$ is multiplied by the spectrum of ambient photons $=n_{v} d N / d(h v)$ (photons $/ \mathrm{cm}^{3}-\mathrm{eV}$ ) and integrated over photon energy it yields a normal cross-section $\left(\mathrm{cm}^{2}\right)$ for absorption. In a kinetic theory this cross-section gives the rate of photon absorption events:

$$
\frac{d N}{d^{3} r d t d(h v)}=n_{I} \int \frac{2 d^{3} p}{h^{3}}|v| f(\varepsilon) \sigma^{a b s} n_{v} \frac{d N_{r}}{d(h v)}
$$

Here $n_{I}$ is the ion number density and $f(\varepsilon)$ is the electron distribution function.

The emission cross-section $\sigma^{\text {emiss }}$ is a differential cross-section to emit a photon of energy $h v$ so its units are $\mathrm{cm}^{2} / \mathrm{eV}$.

$$
\frac{d \sigma^{\text {emiss }}}{d(h v)}=\frac{8 \pi}{3 \sqrt{3}} Z^{2} \alpha^{3}\left(\frac{e^{2} / a_{o}}{\varepsilon_{o}}\right) \frac{a_{o}^{2}}{h v}
$$

To obtain the emission rate, $\sigma^{\text {emiss }}$ is multiplied by $\left(n_{v}+1\right)$ to include stimulated emission and integrated over photon energies (up to the electron's initial energy). The rate of emission of photons of energy $h v$ is (in kinetic theory)

$$
\frac{d N}{d^{3} r d t d(h v)}=n_{I} \int \frac{2 d^{3} p_{o}}{h^{3}}\left|v_{o}\right| f\left(\varepsilon_{o}\right) \frac{d \sigma^{\text {emiss }}}{d(h v)}\left(n_{v}+1\right)
$$

We must adapt these expressions to the different description used in the MD simulation. In that description the electron distribution function $f(\varepsilon)$ is being sampled one collision at a time; the factors $n_{I}$ and $f(\varepsilon)$ are represented by the frequency of attempting the radiation calculation.

The cross-sections become conditional probabilities when we form the ratio to the Coulomb collision cross-section. Analysis of special MD simulations, which did not include pair-interactions, show that the rate of collisions is, as expected from kinetic theory,

$$
n_{e} n_{i}<\sigma v>
$$

where $n_{e}, n_{i}$ are electron and ion number densities, $\sigma$ is the cross-section and $v$ is the relative velocity. For example, if we ask the MD simulation to count the rate at which electrons arrive within a distance $R_{B}$ of an ion, the MD rate is given by the above formula with $\sigma=\pi R_{B}^{2}$ (to about $1 \%$ accuracy).

Given that an electron has arrived within a distance $R_{B}$ of an ion, we take the conditional probability of emission of a photon of energy hv to be the ratio 


$$
\frac{\left\lfloor\frac{d \sigma}{d h v}\left(n_{v}+1\right)\right\rfloor}{\left(\pi R_{B}^{2}\right)}
$$

This conditional probability is proportional to $l / R_{B}{ }^{2}$ but the dependence on $R_{\mathrm{B}}$ cancels because the rate of arrival of the electrons is proportional to $R_{B}^{2}$. We have verified that simulations with different values of $R_{B}$ give similar rates for the radiative events to within the numerical fluctuations.

Our algorithm can now be outlined:

1.) When an electron arrives within a distance $R_{B}$ of an ion, use a random number to decide whether any radiative process occurs. The probability of a radiative process is small so this test is rarely passed. If not, proceed with the usual Coulomb collision. The probability is calculated using the current photon populations in the integrated crosssections.

2.) If there is a radiative event, determine whether it is emission or absorption and determine the photon frequency. In general, emission and absorption do not have equal probabilities according to equations $(19,20)$; however, with equilibrium populations the time-reversed processes would have equal rates. The determination is based on one or several random numbers; the normalized probability distribution is obtained from the cross-sections by the usual Monte Carlo cumulative probability.

3.) The photons are described by photon frequency groups and the emitted/absorbed photon is assigned the energy at the center of the group. This change of photon energy is a discretization approximation and has its largest effect for the highest and lowest photon energies. The electron energy is adjusted to exactly conserve energy.

This algorithm is installed in the Coulomb molecular dynamics code without changing the Ewald sum used to handle the long-range part of the Coulomb interaction. We select photon energy-groups that put primary emphasis on relatively high-energy photons that are expected to be most important for the energetics. The high-energy photons are emitted and absorbed in relatively strong ("close") collisions and these events are expected to be mainly binary electron-ion collisions not strongly influenced by the neighboring ions. It is likely that a different algorithm could be developed to handle the lower photon energies and this algorithm might not need to make the binary-encounter approximation.

The simulations we have performed with the algorithm described here indeed relax to a photon distribution consistent with a Planck function. The low-energy frequency groups relax much more rapidly than the high-energy photons. Simulations performed on a small workstation have substantial numerical noise because the number of photons in a $1000 \mathrm{~A}^{3}$ simulation box is not large at $\mathrm{kT}<5 \mathrm{keV}$. The algorithm has been added to our large parallel simulation code and has been used for large simulations, which give less numerical noise. 
At present the simulations assume the Kramers cross-sections and this is a limitation that precludes study of new effects such as modified emission when an electron simultaneously encounters two nearby ions. We anticipate there should be interference between the emission probabilities and the algorithm described in this section neglects the interference.

The Kramers cross-sections are summed over angular momentum of the incoming electron, although it is evident that the smallest angular momenta are strongly dominant. It would be more accurate to use the angular momentum resolved cross-sections that are given by relatively simple formulas. It would also be possible to improve the accuracy of the Kramers cross-sections by multiplying them by a correction for quantum mechanics, i.e., the Gaunt factor, but this is not a large correction for cases where the cross-section is large.

The algorithm described here has the important advantage that the close collisions, which correspond to electron-nucleus distance shorter than $R_{B}$, are handled as binary collisions and the exact classical Kepler orbits can be used for the Coulomb scattering. These orbits give deflections consistent with the Rutherford cross-section, the same result obtained from a full quantum mechanical solution of the corresponding collision.

In this case there is no need for a QMEP, which inevitably distorts the large-angle collision cross-section, because three-body recombination to negative energy states does not occur in the two-particle classical system. However, if we improved the algorithm for motion at $r<R_{B}$ by including a microfield from the ambient environment, and if this microfield were allowed to change during the collision, then we would again have to confront the problem of recombination. At present we omit the possibility of radiative recombination; this phenomenon will enter in future simulations in which each ion is allowed to be coupled to a collisional-radiative kinetic model.

The calculations described here represent one approach to simulating the coupling of particles and radiation, but other methods or hybrids need to be explored to evaluate all the many physical processes that are present in such a rich environment. We are especially interested in effects of interference in simultaneous collisions of one electron with two or more ions, interference in collisions of two or more electrons with one ion, interference between subsequent collisions, and processes in which the high-frequency quiver velocity of electrons caused by blackbody radiation alters the collision dynamics. For many of these processes there are analytic calculations in the literature but the phenomena interact. It remains to be seen if we can incorporate all these effects in a single simulation. 


\section{6.) MULTICENTER EFFECTS}

In this section we briefly describe our recent efforts to move to an approximate inclusion of multi-center effects in the emission and absorption of radiation. By multicenter effects we mean the additional emission which occurs when two target ions are close together and one electron simultaneously interacts with both, or the additional emission which occurs when two electrons simultaneously interact with the same ion. In each case we compare the multiparticle collision with the sum of the two separate interactions, in the usual spirit of the Virial expansion.

For the multicenter interactions we propose to group together and include interference between the accelerations of the particles involved in the collision. Thus, in an electron-electron collision, if we keep the accelerations of both electrons, which are equal and opposite, we find a small result, zero in the dipole approximation.

An expansion in terms of small clusters of near-neighbor particles may seem to be threatened by the long range of the Coulomb interaction, but in fact the situation is more favorable for radiation than for the plasma equation of state. That is because the emission/absorption probabilities are basically proportional to the square of a Fourier component of the classical acceleration, i.e., $a(\omega)^{2}$, and for Coulomb forces this quantity falls off like $1 / R^{4}$, where $R$ is the particle pair-separation. Of course, the actual convergence with distance is probably much more rapid because the various regions of the plasma are neutral on average so there is strong cancellation of accelerations associated with distant positive and negative charges.

We plan to improve our simulations guided by classical emission calculations for few-particle collisions, using either Lienard-Weichert or normal-mode methods. In this effort we expect to encounter special challenges in finding comparison data: we need quantum or semiclassical emission cross-sections for systems that are not spherically symmetric.

The multicenter calculations predict interference phenomena which have been previously studied using the Born approximation to express the emission/absorption as integrals over the equilibrium pair-correlation functions. [9, 10] Our calculations are effectively non-Born (classical) calculations of the nearest-neighbor part of this interference effect. For specific orientations the interference effects are quite strong but the practical question is what survives averaging over orientations of the local cluster of target particles. 


\section{7.) SIMULATIONS USING THE BINARY-ENCOUNTER APPROXIMATION}

In order to test the radiation algorithm described in Section 6, MD simulations were performed for three-temperature systems of electrons, ions and photons in a cubic simulation box using periodic boundary conditions. The MD was performed with a fully parallel code using a basic leapfrog method [11] with the Coulomb interaction evaluated by an Ewald summation $[12,13]$.

The simulation was performed for a weakly coupled hydrogen plasma with a particle number density of $10^{26} / \mathrm{cm}^{3}$, initial ion temperature of $20 \mathrm{keV}$, electron temperature of $10 \mathrm{keV}$ and radiation temperature of $3 \mathrm{keV}$. The initial particle distributions were obtained by coupling the two species to separate Langevin thermostats, [11] while the radiation initial conditions were selected from a Planckian distribution. The MD results were compared to a multi-group radiation calculation that treats the plasma as a fluid and computes the time evolution of electron and ion temperatures for an LTE plasma undergoing emission and absorption due to bremsstrahlung. The radiation field evolves according to the homogeneous and isotropic semi-classical radiation transport equation, while the electron and ion temperatures evolve according to an energy balance relationship.

Figure 1 shows the evolution of temperature as a function of time compared to the results coming from a code that solves the multi-group radiation equations [20]. The MD simulation assumes nothing about the plasma properties apart from the Coulomb potential; the emission and absorption are governed by the Kramers cross-sections described above. For a hydrogen plasma at the specified conditions, the simulation results are similar to the multigroup radiation calculation.

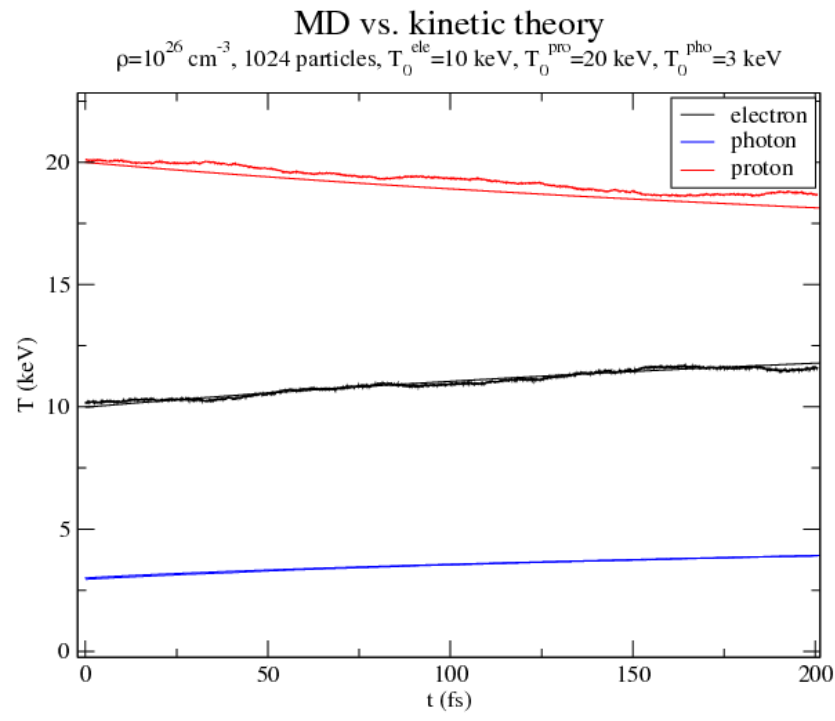

Figure 2: MD and radiation code results for a relaxing non-equilibrium hydrogen plasma. The noisy lines are the MD results. The red line represents the ion temperature, the black line is the electron temperature and the blue line the radiation temperature. 
In order to see the evolution of the radiation field, we enhanced the coupling between photons and electrons by a factor of 100. Figure 2 shows time snap-shots of the evolving radiation intensity from a Planckian at $3 \mathrm{keV}$ for the same three-temperature problem shown in Fig. 2. We see that the low frequency groups are populated earlier as expected for an electron-radiation (Bremsstrahlung) coupling rate $\tau^{-1} \sim 1 / v^{3}$.

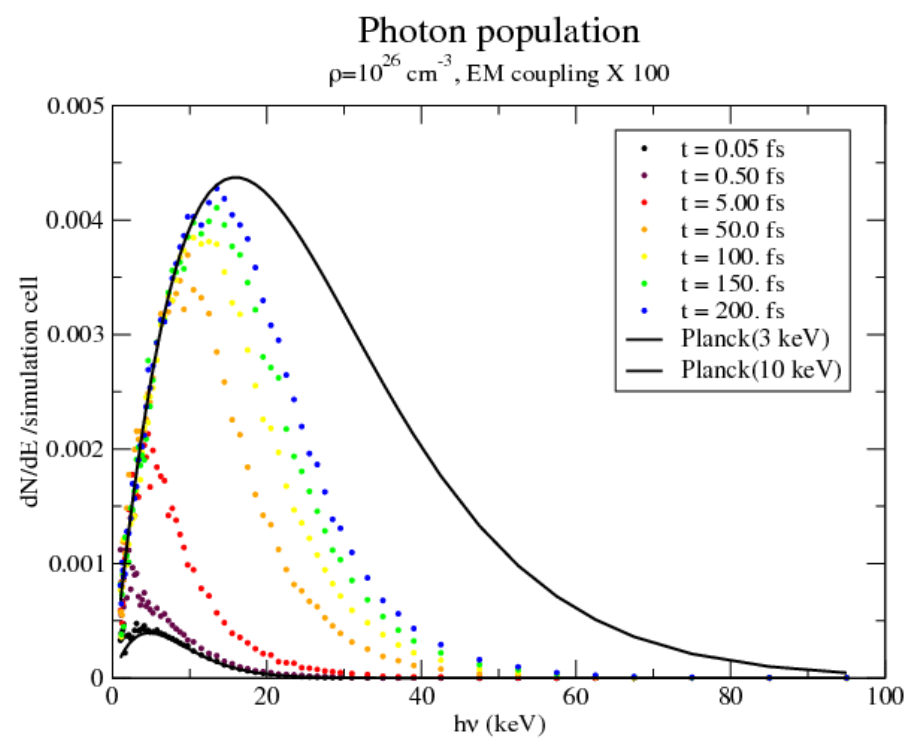

Figure 3: Time snapshots of the radiation specific intensity as a function of photon energy. The smaller black curve represents the initial $3 \mathrm{keV}$ blackbody spectrum. The larger black colred curve is a blackbody at $10 \mathrm{keV}$. The colored dots show time snapshots of the evolving spectrum.

\section{8.) SUMMARY}

The goals of this work are to investigate the effects of impurity ions, fusion products and non-equilibrium kinetic processes in hot plasmas near the threshold for fusion ignition. We will test kinetic-theory models widely used in plasma modeling codes against particle simulations based on the fundamental laws of mechanics and electrodynamics. This work is a natural preparation for upcoming fusion ignition experiments that will be performed on the LLNL NIF laser.

The usual atomic-scale particle simulations of a plasma consider classical pointcharge electrons and ions interacting with instantaneous Coulomb forces. The particles are contained in a simulation box surrounded by periodic images of the instantaneous configuration in the original box. With the development of large parallel computers, this method has come of age and modern simulations involve very large numbers of particles. However, realistic simulations must include radiation phenomena. 
In the work described here, we found it was possible to add a classical EM field to a classical MD particle simulation by two methods: 1.) The Lienard-Weichert solution of the Maxwell equations, and 2.) a normal-mode expansion of classical EM field with a first-order ordinary differential equation for the field amplitudes. Both methods give retardation, magnetic fields, and low-frequency EM wave effects. We have developed codes for both methods and found by both numerical and analytical study of special cases that the Lienard-Weichert and normal-mode solutions agree. This is no surprise because both are in principle exact solutions of the classical Maxwell equations; unfortunately they do correctly relax to the Rayleigh-Jeans radiation field which is physically incorrect for the quantum mechanical description of the radiation. This, of course, is because these are classical theories.

We expect that quantum ingredients are always required to calculate the radiation produced by a hot plasma when the photon energy $h v$ is greater than or equal to the plasma temperature $T$. In this case the classical calculation simply cannot obtain the correct answer. Einstein showed what is needed to get a calculation to relax to the Planck spectrum: absorption and emission must be governed by probabilities whose ratio contains the particle distribution and the usual factors $n_{v},\left(n_{v}+1\right)$.

We have developed a tractable strategy to implement the necessary quantum mechanics at least for radiation from binary electron-ion collisions. The radiation field is represented by an isotropic spectral intensity

$$
I_{v}=\operatorname{chv} n_{v} d N / d h v
$$

The emission and absorption probabilities are obtained from the Kramers cross-sections for emission/absorption, scaled to the Coulomb cross-section to make conditional probabilities for emission/absorption during Coulomb collisions. In this manner we have included only the electron-ion radiation, neglecting small contributions from electronelectron or ion-ion quadrupole emission and Compton scattering.

We performed MC tests to generate the distribution of emission and absorption as weel as to determine the photon frequency. The relative probabilities are obtained from the Kramers cross-sections. It is found that radiation occurs in less than $10^{-3}$ of the strong collision events for the hydrogen plasma studied. Each electron-ion pair gets only one chance to emit or absorb per collision. In this treatment the close collisions are binary and distant particles are neglected during the collision; this approximation is only used when the particles are within $\sim 0.1$ of the average distance between particles. With this approximate method it is possible to do MD simulations including radiative processes. We believe there is a rich variety of microscopic physics to explore using our code: effects of impurities, effects of high-energy particles, e.g., fusion $\alpha$-particles, various types of relaxation to equilibrium, and fusion by high-energy knock-on ions.

ACKNOWLEDGEMENTS: The authors have usefully discussed the work of this paper with a number of scientists, especially including Drs. Takako Kato, Kimitaka Itoh and 
Katsunobu Nishihara, Michael Murillo, John Castor, Jon Weisheit and Lorin Benedict. The work performed under the auspices of the U.S. Department of Energy by Lawrence Livermore National Laboratory under Contract DE-AC52-07NA27344. This work was funded by the Laboratory Directed Research and Development Program at LLNL under project tracking code $09-$ SI-011.

\section{REFERENCES:}

[1] S. Brush, H. Sahlin and E. Teller, J. Chem. Phys. 45, 2102 (1966).

[2] W. H. Butler and W. Kohn, in Electronic Density of States, National Bureau of Standards Special Publication 323, Ed. by L. H. Bennett, US Department of Commerce, Washington DC, 1971.

[3] L. D. Landau and E. M. Lifshitz, Theorie des Champs, Physique Theorique Tome 2, Editions Mir Moscou, 4eme edition. 1989; This book is available in several languages.

[4] N. M. Kroll, "Quantum Theory of Radiation" in Quantum Optics and Electronics, Les Houches, 1964, Ed. by C. de Witt, A. Blandin and C. Cohen-Tannoudji, Gordon and Breach, New York, 1965, p. 1.

[5] C. Cohen-Tannoudji, J. Dupont-Roc and G. Grynberg, Photons and Atoms, WileyInterscience, 1989.

[6] A. Einstein, Physik Z., 18, 121 (1917).

[7] H. A. Kramers, 1923, Phil. Mag. 46, 836.

[8] R. M. More,1991, "Atomic Physics of Laser-Produced Plasmas", in Handbook of

Plasma Physics, vol. 3, Chapter 2; Edited by M. Rosenbluth, R. Sagdeev, A. Rubenchik and S. Witkowski, Elsevier-North Holland, Amsterdam.

[9] See, for example, S. Ichimaru, 1973, Basic Principles of Plasma Physics, W. A. Benjamin, Inc., Reading, Mass.

[10] H. Totsuji, Phys. Rev. A32, 3005 (1985).

[11] Streitz F, Glosli J and Patel M 2006 Phys. Rev. Lett. 96225701.

[12] Ewald P 1921 Ann. Phys. 64, 253.

[13] DeLeeuw S, Perram J and Smith E 1980 Proc. R. Soc. London, Ser. A 373, 27.

[14] J. Glosli, F. Graziani. R. More, M. Murillo, F. Streitz and M. Surh, J. Phys. A: Math. Theor. 42214030 (2009).

[15] R. M. Shagaliev, 2006, "Finite Difference Methods Implemented in SATURN Complex to Solve Multi-Dimensional Time-Dependent Transport Problems", in

Computational Methods in Transport; Edited by F. R. Graziani, Springer, BerlinHeidelberg-New York.

[16] M. M. Marinak, G. D. Kerbel, N. A. Gentile, O. Jones, D. Munro, S. Pollaine, T. R. Dittrich and S. Haan, 2001 Phys. of Plasmas 8, 2275

[17] G. Kelbg, 1963 Ann. Phys. 12, 219

[18] T. Dunn and A. A. Broyles, 1967 Phys. Rev. 157, 156

[19] C. S. Jones and M. S. Murillo, 2007 High Energy Density Phys. 3, 379

[20] F. Graziani, 2006 "Radiation Diffusion; Numerical and Physical Concepts" in Open Issues in Core Collapse Supernova Theory (Proceedings from the Institute for Nuclear Theory); Edited by A Mezzacappa, World Scientific Publishing, Singapore 
[21] A. A. Correa, L. X. Benedict, D. A. Young, E. Schwegler, and S. A. Bonev, 2008 Phys. Rev B 78, 024101 